\title{
Don't Judge a Book by Its Cover: A Clinical Neuropathological Conference Case
}

\author{
Foziah Alshamrani, Doug Zochodne, Kristopher Langdon, Sachin Pandey (1), \\ Lee Cyn Ang, Keith MacDougall, David MacDonald, Sarah A. Morrow
}

Keywords: Tumefactive demyelinating lesions (TDL), Glioblastoma multiforme, Multiple sclerosis, Acute disseminated encephalomyelitis (ADEM), MRI, Histopathology

doi:10.1017/cjn.2020.21

Can J Neurol Sci. 2020; 47: 404-409

\section{Case Presentation: Dr. Foziah Jabbar Alshamrani}

A 58-year-old right hand dominant woman known to have hypertension, type 2 diabetes, and dyslipidemia presented to the multiple sclerosis (MS) clinic in 2017 for evaluation of a brain lesion, thought to be acute disseminated encephalomyelitis (ADEM). She was previously well, beyond a history of 2 days of lower facial numbness at age 30 that was not investigated.

In December 2017, following an upper respiratory tract infection, she noticed slurred speech and right facial weakness. She had a left frontal lobe hypodense lesion on her initial head CT (Figure 1), confirmed by a subsequent brain MRI (Figure 2). She denied other neurological symptoms including visual changes, sensory loss, sphincter dysfunction, or L'hermitte's sign. Initial blood work was normal including serum angiotensin-converting enzyme (ACE), serum protein electrophoresis, antinuclear antibody, anti-double stranded (ds) DNA, and Lyme serology. Her urinalysis was normal. A lumbar puncture demonstrated normal cerebrospinal fluid (CSF) protein, glucose, and negative oligoclonal bands (OCBs).

She was treated with three daily doses of $1000 \mathrm{mg}$ IV methylprednisolone, 3 months after symptom onset, with improvement of her dysarthria and facial weakness. After 1 month, she developed new gait ataxia, difficulty with language fluency, and fatigue. When assessed in the MS clinic, her repeat MRI noted the frontal lobe lesion was smaller following methylprednisolone (Figure 3).

There was no family history of MS or other inflammatory neurological disorders. On examination, general physical examination was normal. Her neurologic findings included spastic dysarthria with slowed speech, impaired word finding with normal repetition and comprehension. Visual acuity, fundoscopy, pupillary reflexes, and eye movements were normal. There may have been borderline hip flexor weakness, but motor coordination, deep tendon reflexes, and plantar responses were otherwise normal. Sensation, gait, and stance were intact.

\section{Discussion: Dr. Doug Zochodne}

Thank you. An important step in sorting through this problem is to essentially ignore the route of referral the patient experienced and consider her story and findings as a "clean slate." Simply by being referred to an MS clinic does not mean that this is what the problem may be related to. In clarifying the localization, the dysarthria and perhaps the word finding difficulty fits with her known imaging lesion, as would the history of gait dysfunction. However, there were no evident gait abnormalities on examination. The apparent proximal leg weakness does not really fit unless we are thinking of a second neurological problem such as a symmetrical myopathy, perhaps related to her glucocorticoid use, although it was not prolonged. Knowing if there was subtle neck flexor weakness, proximal upper limb weakness or difficulty arising from a squat would support that possibility but these were not apparently noted. Bilateral convexity lesions can cause symmetrical leg weakness but in the setting of spasticity, other upper motor neuron signs, and bladder dysfunction. A more likely scenario often seen is that "borderline" hip flexor weakness is an artifact of our examination, more likely to occur in someone with a large body habitus or hip discomfort. It is important not to grade muscles as $4 / 5$ if weakness is only borderline and not pathological. I understand there was no proximal sensory loss in the legs. More relevant to the case was the absence of a history of a recent infection, fever, headache, or stiff neck that might support the referral diagnosis of ADEM. The pace of onset of these problems was subacute, against a vascular cause. I did have the opportunity before today's discussion to review the unenhanced $\mathrm{CT}$ which showed a juxtacortical hypodense lesion but with abnormal somewhat thickened appearing grey matter overlying it. This is not in a vascular territory. The density is not low enough to suggest a large cyst and I do not see calcification to suggest oligodendroglioma. Mass effect was not prominent on the image I had to review, and there were no other obvious lesions.

From the Neurology Department King Fahad University Hospital, Imam Abdulrahman Bin Faisal University, Dammam, Saudi Arabia (FA); Department of Clinical Neurological Sciences, University of Western Ontario (Western), ON, Canada (FA, KM, DM, SAM); Department of Medicine, Division of Neurology, University of Alberta, AB, Canada (DZ); Department of Pathology and Laboratory Medicine, University of Western Ontario (Western), ON, Canada (KL, LCA); Department of Medical Imaging, University of Western Ontario (Western), ON, Canada (SP)

Received July 20, 2019. Final Revisions Submitted January 18, 2020. Date of ACCEPTANCE January 25, 2020.

Correspondence to: Sarah A. Morrow, Department of Clinical Neurological Sciences, University of Western Ontario (Western), ON, Canada. Email: Morrow@lhsc.on.ca 


\section{Case Presentation: Dr. Alshamrani}

Investigations showed a normal complete blood count, urea, creatinine, calcium, magnesium, electrolytes, liver enzymes, glucose, thyroid stimulating hormone, ACE, serum protein electrophoresis, antinuclear antibody, anti-ds DNA, and anti-extractable nuclear antigen screen. Her serum anti-neuromyelitis optica and anti-myelin oligodendrocyte glycoprotein antibodies were negative.

LP was repeated: WBC: 6, RBC: 75, lymphocytes: $62 \%$, glucose: 5.5 (serum 4.7), protein: 0.44 , G. stain: negative, IgG index: 61. CSF was negative for ACE and OCB. CT of her chest, abdomen, and pelvis was normal. Mammography demonstrated only benign changes. Visual-evoked potentials were normal.

In June 2018, she presented to the emergency department with recurrence of right facial asymmetry, dysarthria, and an episode of right facial twitching. She was diagnosed with focal onset seizures without loss of awareness (simple partial epilepsy) and was treated with levetiracetam $500 \mathrm{mg}$ twice daily. MRI brain was repeated, demonstrating growth in the lesion.

\section{Discussion: Dr. Doug Zochodne}

The differential diagnosis of this lesion includes several entities. Somewhat against but not excluding a large tumefactive MS lesion is the abnormal appearing overlying grey matter and prominent partial seizures; the probable focal seizure suggests cortical involvement, beyond white matter alone. She did not have a typical history to suggest ADEM. The enhancing MR lesion did diminish in size after her course of steroid. This would support the possibility of a lymphoma, which I have put high on the list of possibilities or an inflammatory lesion. In my experience, primary CNS lymphomas are often superficial or periventricular, deeper in the white matter, sometimes multiple and have not seemed as variegated as this, but with much more intense and uniform enhancement. This is supported by Haldorsen et al. ${ }^{1}$ who pointed out that primary CNS lymphomas are hypo- or iso-intense on T1 MR and iso- to hyper-intense on T2 MR and show moderate to marked, usually homogenous contrast enhancement. Ring enhancement can occur but is far less common and edema not as marked as with glioma. The current case may have fit the intensity characteristics but it was variegated, had ring enhancement and prominent edema. The disappearance of primary CNS lymphoma with corticosteroid therapy can be dramatic and near complete. Somewhat concerning is that persistence of impressive edema in this case. I think a metastatic lesion is unlikely but not impossible. Other less common possibilities might include a focal deposit of sarcoidosis, inflammatory cysticercosis, or a tubercular, spirochaete, or fungal lesion. One could not exclude a glioma given the variation in density and the cortical changes but I think it must be unusual for gliomas to improve to this degree on imaging studies. Most clinicians do see patients improve, with resolution of signs when dexamethasone is added at the outset of therapy. However, in my experience, this has been associated with somewhat less enhancement, less edema but probably no change in the main lesion. This patient requires an urgent neurosurgical biopsy.

\section{Neuroradiology: Dr. Sachin Pandey}

Initial CT scan of the head (Figure 1) revealed a focal, intraaxial, left frontal hypodensity, primarily juxtacortical in location.

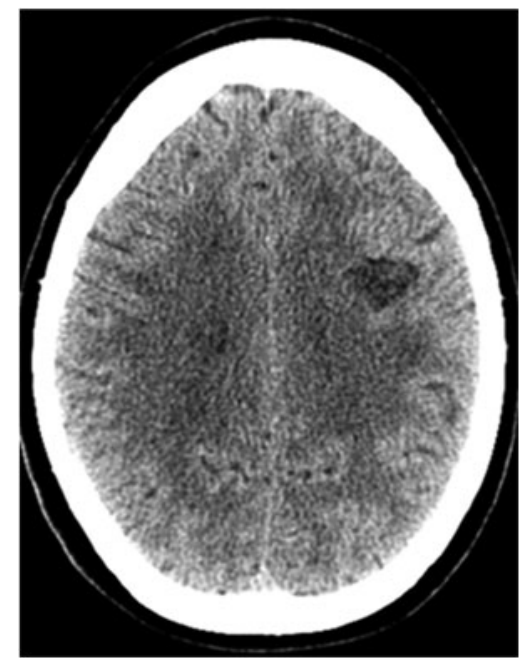

Figure 1: Initial brain CT scan axial section showing left frontal lobe hypodensity.

A subsequent brain MRI (January 2018) confirms the left frontal lesion which was primarily hypointense on T1W images (Figure 2A), demonstrating a heterogenous, primarily peripheral pattern of enhancement (Figure 2B). Both Figures 3 and 4 demonstrate changes in the appearance of the lesion over time, namely prior to, and following, cortiocosteroid therapy. Figure 3 demonstrates a dramatic decrease in the size of the enhancing component of the lesion, notably, however, without total resolution of the abnormal enhancement. Conversely, T2-FLAIR images over that same time period (Figure 4) continue to demonstrate a disproportionately large area of surrounding abnormal signal hyperintensity, which decreased in size very minimally. Overall, the initial imaging considerations would be quite broad, favoring a moderate or high-grade glial neoplasm, metastatic deposit, or tumefactive process. Other neoplastic entities such as CNS lymphoma would be less likely given the peripheral location, lack of uniform enhancement, and relative hypodensity on baseline CT. The dramatic decrease in the size of the enhancing component of lesion following steroid therapy did serve to refine the list of diagnostic considerations, namely rendering a tumefactive demyelinating process as being more likely. However, the possibility of a high-grade neoplastic process persisted, particularly with the absence of corresponding improvement of the area of FLAIR signal abnormality with corticosteroid administration.

\section{Neurosurgery: Dr. KeIth MacDougall}

This patient was referred to me with the working diagnosis of primary CNS lymphoma versus tumefactive demyelinating lesion (TDL). Lymphoma was the main consideration given the previous excellent response to systemic methylprednisolone. A demyelinating process seemed unlikely because of the history of a focal motor seizure but was still a primary consideration. We discussed performing a frame-based stereotactic biopsy with the patient and she agreed. The biopsy was technically straightforward and four cores were provided for pathology. Frozen section on the tissue suggested high-grade glioma. 


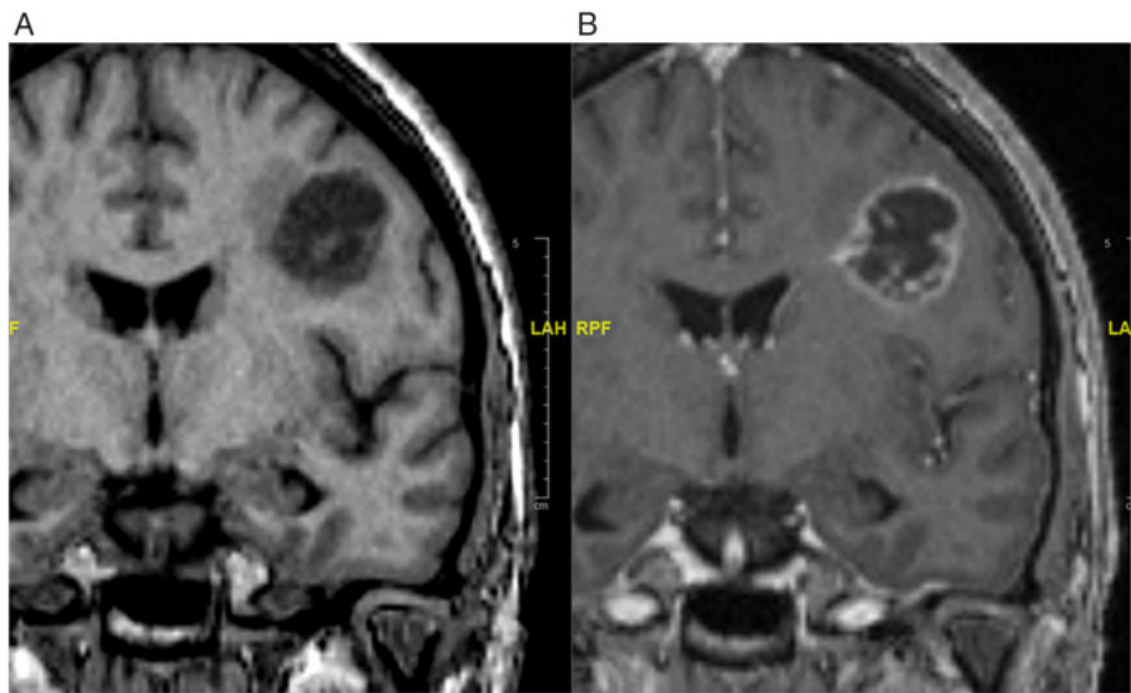

Figure 2: (A) Coronal pre-contrast T1W image from the baseline MRI assessment demonstrating a predominantly hypo-intense, intra-axial, left frontal lesion, largely centered in the peripheral white matter. (B) Coronal post-contrast T1W image demonstrating heterogeneous, predominantly peripheral enhancement of the same lesion.

A

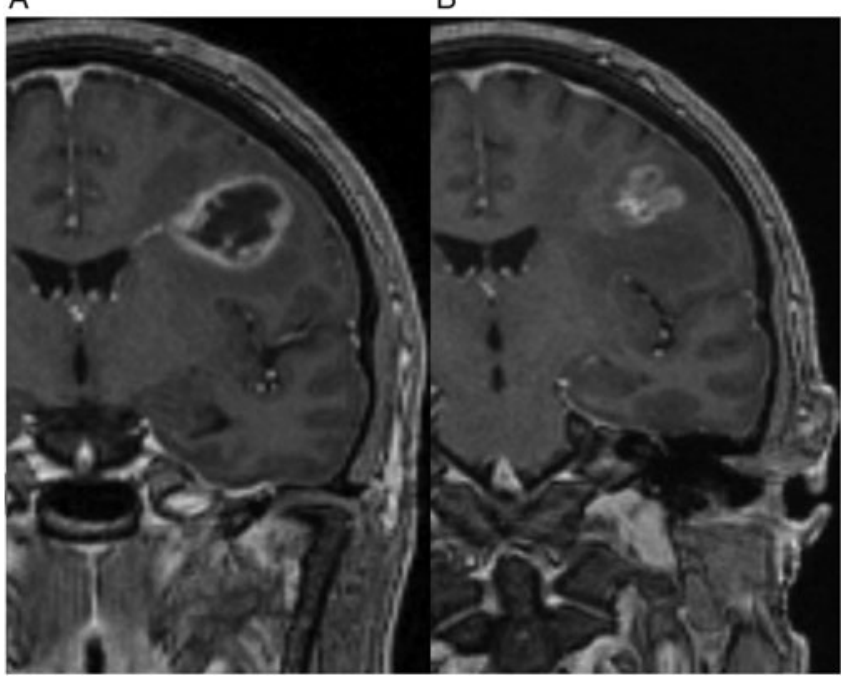

Figure 3: Coronal T1W post-contrast images in Feb (A) vs. May (B) 2018. These are acquired prior to, and then following, steroid therapy, respectively. There is a notable decrease in the size of the enhancing component of the lesion.

\section{NeUropathology: Dr. KRistopher Langdon}

Pathological assessment of the submitted tissue revealed a hypercellular, pleomorphic neoplasm with a fibrillar background without any obvious architectural pattern. The neoplastic cells had varying amounts of eosinophilic to clear cytoplasm, primarily with indistinct cellular borders. Some of the neoplastic cells appeared "epitheloid." The nuclei were atypical and contained coarse chromatin and often distinct nucleoli. There was brisk mitotic activity, foci of necrosis, and microvascular proliferation. The neoplastic cells stained positive for MAP2 with the background fibrillar processes staining immunopositive for GFAP. ATRX was nuclear positive (not mutated) with approximately
20-30\% of nuclei staining positive for p53. Molecular genetics (next generation sequencing) did not identify any mutation in the IDH1, IDH2, and BRAF (V600) genes. Cytogenetic analysis (fluorescent in situ hybridization) revealed amplification of epidermal growth factor receptor and polysomy of chromosome 7. The histological and immune-histomorphological features, combined with the results of the molecular studies, support the diagnosis of glioblastoma, IDH-wild-type (WHO Grade IV) Figure 5.

\section{Discussion: DR. ZOCHODNE}

I think this was an instructive patient given the unusual clinical course and apparent partial resolution of imaging characteristics secondary to glucocorticoid use. It reinforces a maxim of the late Miller Fisher who once told us (paraphrased) that case series generated misinformation because they ignore the individuality of patients and their presentations; arguing that $n=1$ was a more honest approach to clinical work. There were red flags in retrospect given the gray matter changes, seizures, prominent edema, and atypical appearance for lymphoma. What the case also reinforces is that imaging, so frequently relied on exclusively in modern neurology, is only a shadow of the complex biology of tissues targeted by disease. One might speculate on whether a prominent inflammatory reaction treated with glucocorticoids accounted for some of its appearance. If so, how do glioma tumor cells generate this response in nearby brain? A final point is that given better availability of MR imaging over the past two decades, getting serial scans done prior to a definitive biopsy is easier and it may be that these kinds of changes in tumor imaging are more common than we realize.

\section{Neuro-Oncology: Dr. David MacDonald}

Following stereotactic brain biopsy, the patient had persistent and progressive dysarthria and word finding difficulties. She was assessed in the neuro-oncology clinic; treatment with radiation 


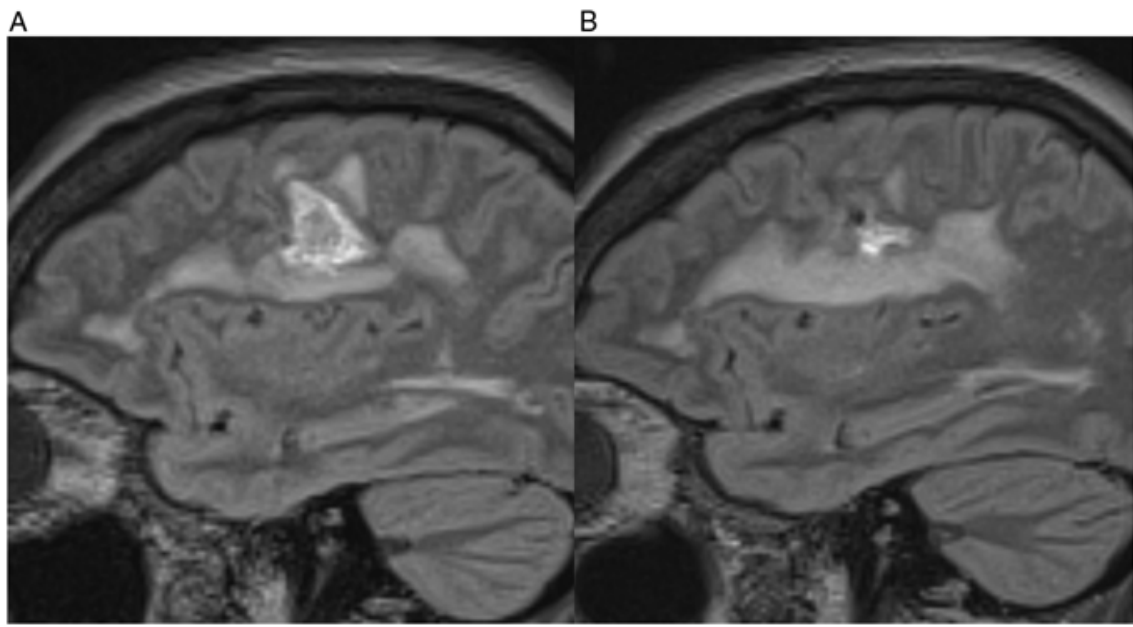

Figure 4: Sagittal T2-FLAIR images in Feb (A) vs. May (B) 2018. These are acquired prior to, and then following, steroid therapy, respectively. There is only very mild decrease in the extent of FLAIR signal abnormality.
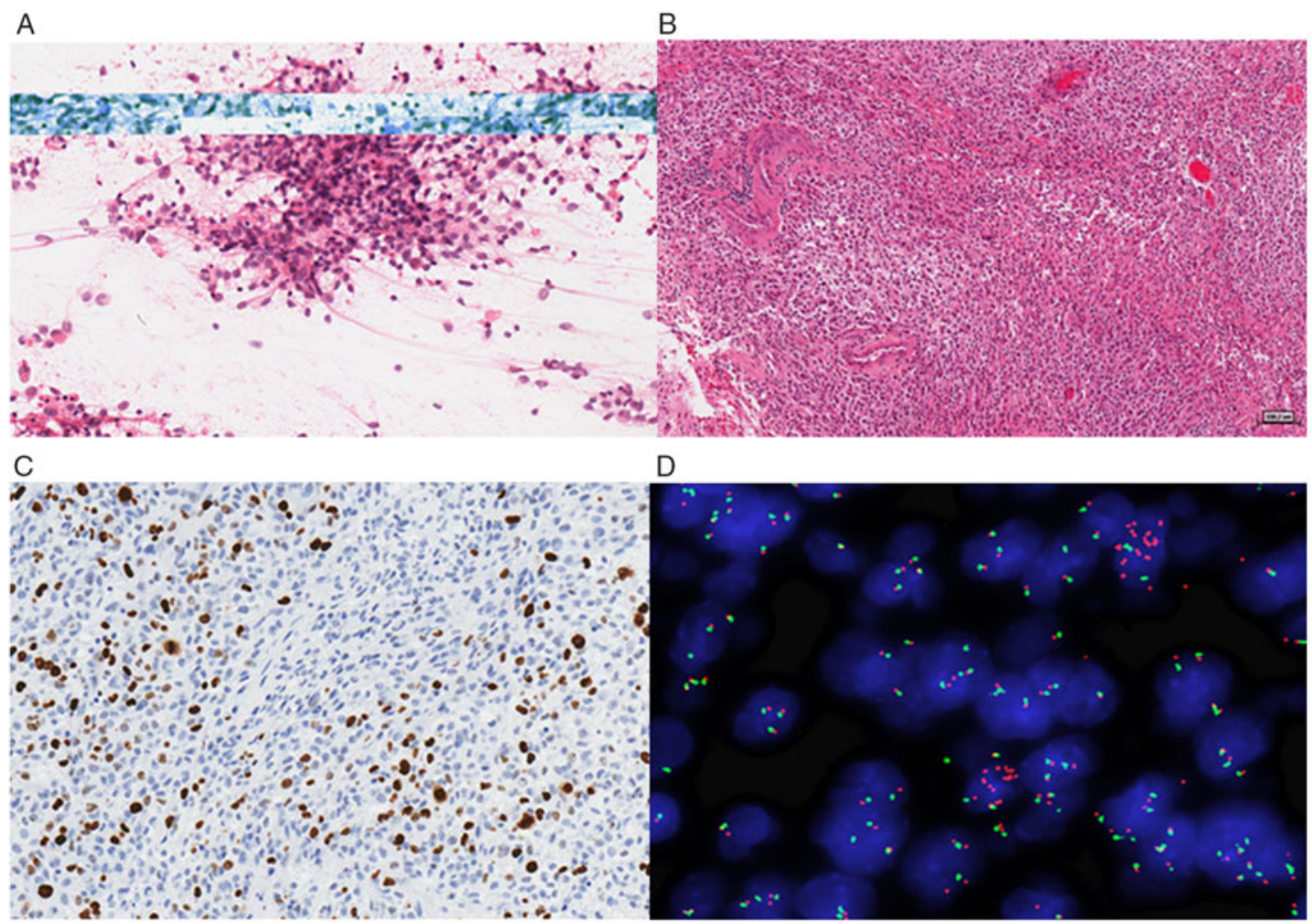

Figure 5: (A) Smear preparation at the time of frozen sectioning demonstrates cellular pleomorphism, nuclear atypia, and fibrillar processes. (B) H\&E stain of the left frontal lesion showed a hypercellular, pleomorphic neoplasm on a fibrillar background that failed to arrange in any particular pattern. The neoplastic cells have varying amounts of eosinophilic to clear cytoplasm, primarily with indistinct cellular borders. Some of the neoplastic cells appear "epitheloid." Brisk mitotic activity, foci of necrosis, and microvascular proliferation are seen. (C) Ki-67 staining highlights the brisk proliferative activity. (D) Fluorescent in situ hybridization for probes against the EGFR gene (red) and chromosome 7 centromere (green) demonstrate EGFR amplification. 
therapy and chemotherapy with temozolomide and dexamethasone was initiated.

\section{Topic Review: Dr. Alshamrani}

One of the rare variants of demyelinating disease is TDLs. ${ }^{2}$ There is no clear definition of TDL; however, an acceptable definition is a demyelinating lesion that is greater than $2 \mathrm{~cm}$ in diameter that may or may not have perilesional edema, mass effect, and/or ring enhancement. ${ }^{3}$ TDL has a peculiar presentation in comparison to classic MS. TDL often manifests with signs and symptoms of space occupying lesion with new onset cortical signs, such as seizures that can occur in $6 \%$ of cases. ${ }^{4}$ The clinical presentation of glioblastoma multiforme (GBM) may also mimic TDL. However, our patients' age at presentation is more in keeping with neoplasm than TDL and MS, and makes ADEM - as the main reason for referral in this case unlikely clinically and radiologically based on the diagnostic criteria as it usually produces a widespread central nervous system disturbance, often with impaired consciousness and/or encephalopathy, and it is one of the required clinical features for a diagnosis of ADEM. Also, lesion characteristics on MRI for ADEM are large multifocal lesions in the supratentorial or infratentorial white matter. Rarely, brain MRI shows a single lesion like our patient MRI here. ${ }^{5,6}$ Furthermore, she complained of worsening motor symptoms and later on had an episode of seizure, indicating progression and cortical involvement, as noted by Dr. Zochodne.

Early dramatic response to corticosteroids is often seen, although temporary, in GBM and other neoplastic lesions such as CNS lymphoma secondary to perilesional edema improvement. ${ }^{7}$ In our case, the response was unusual in that an improvement was not seen in the FLAIR hyperintense (edema) component of the lesion but rather in the enhancing component.

Neuroimaging plays a critical role in differentiating TDL from other primary brain tumors like GBM. It is reported that TDLs have a predilection toward the frontal and parietal lobes. ${ }^{4}$ TDLs classically are well-circumscribed lesions that are supratentorial and often fail to fulfill Barkhof MS criteria. ${ }^{4,8}$ Two studies reported better diagnostic performance when combining conventional MR imaging and advanced MR imaging. ${ }^{9,10}$ There was an attempt done to classify TDLs based on the MRI features into: megacystic, Balo-like, infiltrative, and ring-like. ${ }^{11}$ Accordingly, the conventional MR imaging findings of TDLs were recorded as follows: (1) an open ring or incomplete rim enhancement, (2) a closed ring or complete rim enhancement, (3) a T2 hypointense rim, (4) an absent or mild mass effect, and (5) absent or mild perilesional edema. Among those MR imaging characteristics, one of the highly specific MR imaging features according to a meta-analysis and systemic review by Suh et al. was an open ring or incomplete rim enhancement that favored TDL over primary brain tumor like GBM. ${ }^{12}$ TDL overall has less cerebral blood volume compared to brain neoplasms. ${ }^{13-15}$ Another striking feature to distinguish TDLs from GBM is the regions of hypo-attenuation on non-contrast CT which were previously enhanced on MR imaging seen in TDLS while calcification or hemorrhage seen more with GBM. ${ }^{16}$ Diffusion-weighted imaging (DWI) showed peripheral diffusion restriction in TDLs ${ }^{14,15}$ and higher diffusion coefficient compared to GBM. ${ }^{9}$
Finally, presence of OCBs in the CSF could provide an additional reassurance in favor of TDLs rather than GBM which absent in our case and lead to performing biopsy.

Our patient worsened shortly after her improvement with corticosteroids and without close observation she might have continued to be mislabeled with the diagnosis of TDL and missed the opportunity for further definitive workup. In order to avoid misdiagnoses of TDLs with other vanishing tumors, a suggested approach would be close monitoring of patients following corticosteroid therapy. Fortunately, upon further investigations, a conclusive diagnosis of GBM was reached, although it is an unfortunate diagnosis.

\section{Conclusion}

This case illustrates the challenges in the diagnosis of TDLs and brain neoplasms, as well as the need to ensure that soft signs (such as a previous history of facial palsy) do not bias the potential diagnostic process. It raises the importance of re-addressing a potential diagnosis if there are red flags, if a diagnosis of MS would be unusual, such as in older patients, if there is no previous history of MS, if there is inconclusive or suspicious imaging, and if CSF is negative for OCB. Pathological diagnosis is essential in such situations.

\section{Disclosures}

FA, DZ, KL, SP, LCA, KM, and DM have no disclosures.

SAM has, in the past 3 years, served on advisory boards for Biogen Idec, EMD Serono, SanofiGenzyme Canada, Novartis, Roche, has received Investigator Initiated Grant Funds from Biogen Idec, Novartis, Roche, and has acted as site PI for multi-center trials funded by Novartis, Genzyme, Roche, Adamas, and AbbVie.

\section{Statement of Authorship}

FA: CASE PRESENTATION, topic review and manuscript writing. DZ: CASE discussion, manuscript writing, and final revision. KL: Neuropathology discussion and writing. SP: Neuroradiology discussion and writing. LCA: Neuropathology discussion and writing. KM: Neurosurgery discussion and writing. DM: Neuro-oncology discussion and writing. SAM: CNPC main coordinator, manuscript writing, manuscript revision, and final approval.

\section{REFERENCES}

1. Haldorsen IS, Espeland A, Larsson EM. Central nervous system lymphoma: characteristic findings on traditional and advanced imaging. AJNR Am J Neuroradiol. 2011;32(6):984-92.

2. Poser S, Lüer W, Bruhn H, Frahm J, Brück Y, Felgenhauer K. Acute demyelinating disease: classification and non-invasive diagnosis. Acta Neurol Scand. 1992;86(6):579-85.

3. Abdoli M, Freedman M. Neuro-Oncology dilemma: tumour or tumefactive demyelinating lesion. Mult Scler Relat Disord. 2015;4(6):555-66.

4. Lucchinetti CF, Gavrilova RH, Metz I, et al. Clinical and radiographic spectrum of pathologically confirmed tumefactive multiple sclerosis. Brain. 2008;131(Pt 7):1759-75.

5. Koelman DL, Chahin S, Mar SS, et al. Acute disseminated encephalomyelitis in 228 patients: a retrospective, multicenter US study. Neurology 2016; 86:2085. 
6. de Seze J, Debouverie M, Zephir H, et al. Acute fulminant demyelinating disease: a descriptive study of 60 patients. Arch Neurol 2007; 64:1426.

7. Bromberg JE, Siemers MD, Taphoorn MJ. Is a 'vanishing tumor' always a lymphoma?. Neurology. 2002; 59(5):762-4.

8. Altintas A, Petek B, Isik N, et al. Clinical and radiological characteristics of tumefactive demyelinating lesions: follow-up study. Mult Scler. 2012; 18(10):1448-53.

9. Mabray MC, Cohen BA, Villanueva-Meyer JE, et al. Performance of apparent diffusion coefficient values and conventional MRI features in differentiating tumefactive demyelinating lesions from primary brain neoplasms. AJR Am J Roentgenol. 2015;205:1075-85.

10. Hiremath SB, Muraleedharan A, Kumar S, et al. Combining diffusion tensor metrics and DSC perfusion imaging: can it improve the diagnostic accuracy in differentiating tumefactive demyelination from high-grade glioma?. AJNR Am J Neuroradiol. 2017;38(4):685-90.

11. Seewann A, Enzinger C, Filippi M, et al. MRI characteristics of atypical idiopathic inflammatory demyelinating lesions of the brain: a review of reported findings. J Neurol. 2008;255:1-10.
12. Suh $\mathrm{CH}$, Kim HS, Jung SC, Choi CG, Kim SJ. MRI findings in tumefactive demyelinating lesions: a systematic review and meta-analysis. AJNR Am J Neuroradiol. 2018; 39(9):1643-49.

13. Cha S, Pierce S, Knopp EA, et al. Dynamic contrast-enhanced t 2 *weighted MR imaging of tumefactive demyelinating lesions. AJNR Am J Neuroradiol. 2001;22(6):1109-16.

14. Kilic AK, Kurne AT, Oguz KK, et al. Soylemezoglu F, Karabudak R. Mass lesions in the brain: tumor or multiple sclerosis? Clinical and imaging characteristics and course from a single reference center. Turk Neurosurg. 2013;23:728-35.

15. Sánchez P, Meca-Lallana V, Barbosa A, Manzanares R, Palmí I, Vivancos J. Tumefactive demyelinating lesions of 15 patients: clinico-radiological features, management and review of the literature. J Neurol Sci. 2017;381:32-38.

16. Kim DS, Na DG, Kim KH, et al. Distinguishing tumefactive demyelinating lesions from glioma or central nervous system lymphoma: added value of unenhanced CT compared with conventional contrast-enhanced MR imaging. Radiology. 2009;251(2):467-75. 